\title{
Unstable wide complex tachycardia: Consider hyperkalemia while preparing for cardioversion
}

\author{
2016 \\ Lindsay Leech and Julie Welch \\ Indiana University School of Medicine
}

A 67-year-old male presented to the emergency department with a chief complaint of weakness and abdominal pain. The patient was a resident of an extended care facility where he had lived for the past four months after extensive resection of an abdominal sarcoma complicated by wound dehiscence and an enterocutaneous fistula. Additional past medical history included type II diabetes mellitus, hypercalcemia, hypertension and chronic kidney disease stage II (CKD). On initial presentation the patient was afebrile, diaphoretic, tachycardic (heart rate 120-170's) with a labile systolic blood pressure (down to $60 \mathrm{mmHg}$ ). He was awake, alert, and cachectic, in moderate distress, with extremely dry mucus membranes, cracked lips, and no urine output. His abdomen showed a healing surgical wound with wet to dry dressings and a small fistula. An emergent electrocardiogram (EKG) was obtained ( Image 1 ) and labs were sent. His EKG showed a wide complex tachycardia (QRS 150, QTc 551) at a rate of 162. Due to these EKG findings and low blood pressure, the patient was deemed an unstable wide-complex tachycardia and preparation for cardioversion was initiated. However, while the patient was being placed on the cardioversion monitor with electrode pads, additional causes of his EKG findings were considered, including hyperkalemia. With his history of CKD, no urine output and hypercalcemia, immediate treatment with IV sodium bicarbonate was initiated. His rhythm strip narrowed in response and a second dose was given. A repeat EKG ( Image 2 ) revealed a narrow-complex junctional tachycardia (QRS 106, QTc 464) at a rate of 105. His blood pressure improved to systolics in the 120's.

This is the author's manuscript of the article to be published in final edited form as:

Leech, L., \& Welch, J. (2016). Unstable wide complex tachycardia: Consider hyperkalemia while preparing for cardioversion. Visual Journal of Emergency Medicine, 5, 15-16.

https://doi.org/10.1016/j.visj.2016.04.005 
Laboratory findings confirmed the diagnosis of hyperkalemia and acute renal failure. The patient had an elevated potassium of $7.2 \mathrm{mmol} / \mathrm{L}$, an elevated creatinine of $4.4 \mathrm{mg} / \mathrm{dL}$ (his baseline was $1.6 \mathrm{mg} / \mathrm{dL}$ ) and a normal calcium of $8.6 \mathrm{mg} / \mathrm{dL}$. Further treatment for hyperkalemia was initiated with IV calcium

gluconate, IV insulin with IV 50\% dextrose, and nebulized albuterol. A foley catheter was placed without difficulty to ensure there was not a urethral obstruction contributing to the renal failure and to follow his urine output. Nephrology was emergently consulted for hemodialysis and the patient was admitted to the ICU. 
Figure 1. Patient's EKG upon arrival to the emergency department.

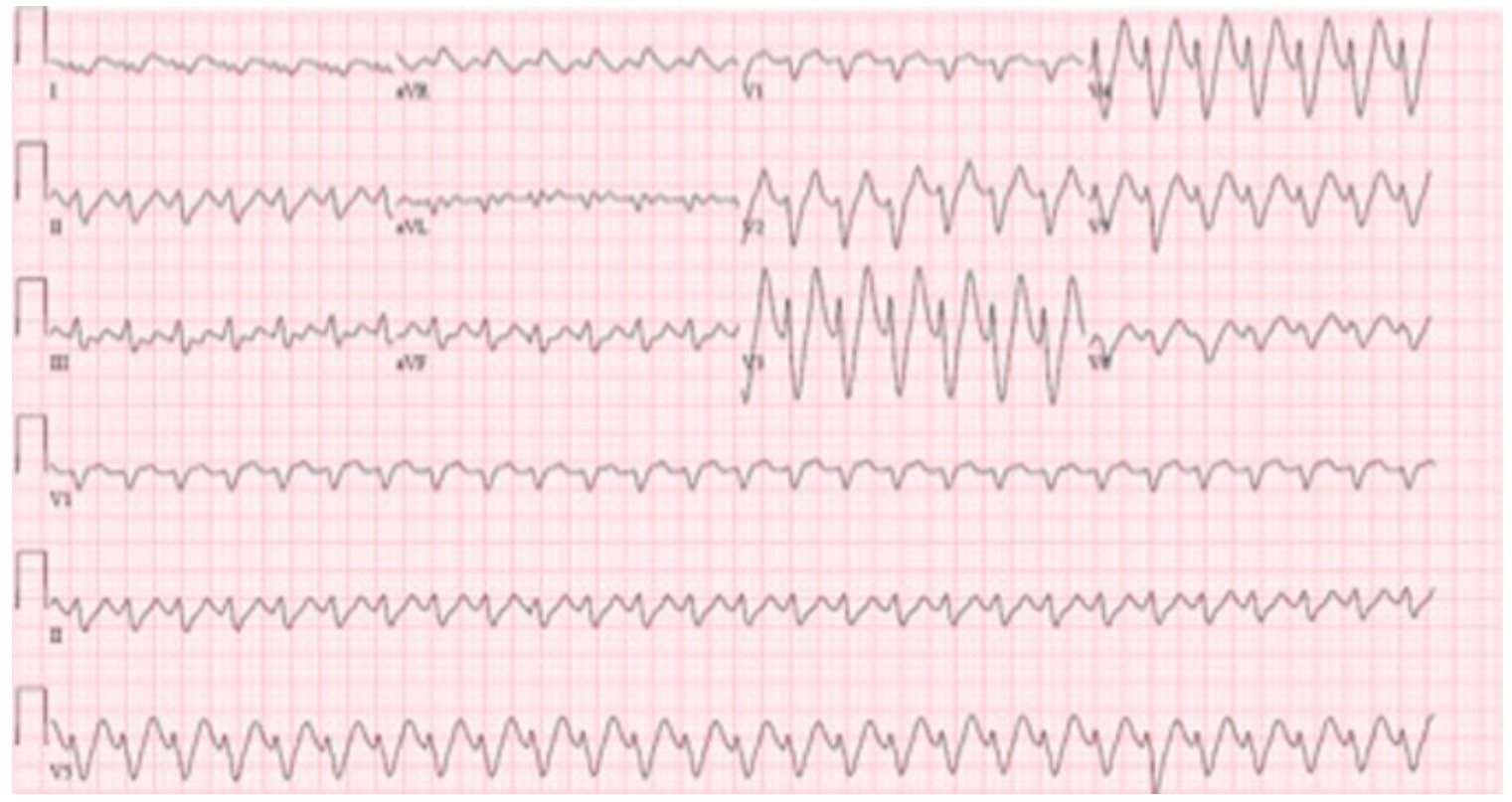


Figure 2. Patient's EKG after receiving IV sodium bicarbonate.

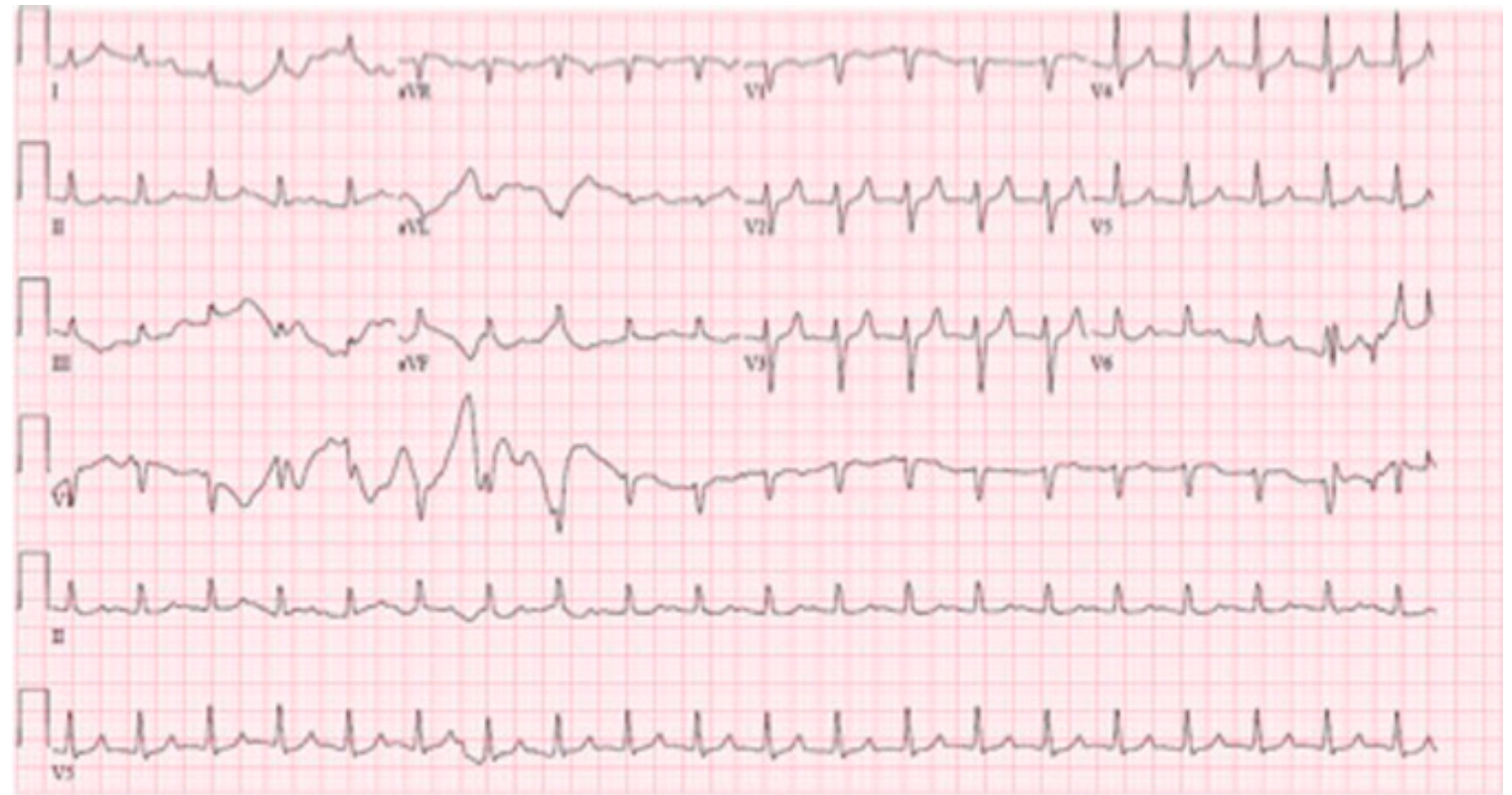

